University of Wollongong

Research Online

Faculty of Social Sciences - Papers (Archive) Faculty of Arts, Social Sciences \& Humanities

2016

Same-day use of opioids and other central nervous system depressants amongst people who tamper with pharmaceutical opioids: A retrospective 7-day diary study

Amy Peacock

University of New South Wales

Raimondo Bruno

University of Tasmania

Briony K. Larance

University of Wollongong, blarance@uow.edu.au

Nicholas Lintzeris

University of Sydney

Suzanne Nielsen

University of New South Wales

See next page for additional authors

Follow this and additional works at: https://ro.uow.edu.au/sspapers

Part of the Education Commons, and the Social and Behavioral Sciences Commons

Research Online is the open access institutional repository for the University of Wollongong. For further information contact the UOW Library: research-pubs@uow.edu.au 


\title{
Same-day use of opioids and other central nervous system depressants amongst people who tamper with pharmaceutical opioids: A retrospective 7-day diary study
}

\begin{abstract}
Objective The aims were to determine: (i) quantity and frequency of same-day use of opioids with benzodiazepines and/or alcohol amongst people who regularly tamper with pharmaceutical opioids; and (ii) socio-demographic, mental health, harms and treatment profile associated with same-day use of high doses. Method The cohort $(n=437)$ completed a retrospective 7-day diary detailing opioid, benzodiazepine, and alcohol intake. Oral morphine equivalent (OME) units and diazepam equivalent units (DEU) were calculated, with $>200 \mathrm{mg} \mathrm{OME,}>40 \mathrm{mg}$ DEU and $>4$ standard alcoholic drinks (each $10 \mathrm{~g}$ alcohol) considered a "high dose". Results One-half (47\%) exclusively consumed opioids without benzodiazepines/alcohol; $26 \%$ had days of opioid use with and without benzodiazepines/alcohol; and $26 \%$ always used opioids and benzodiazepines/alcohol. Same-day use of opioids with benzodiazepines/ alcohol typically occurred on 1-3 days in the past week. Six in ten (61\%) participants reported high dose opioid use on at least one day; one in five (20\%) reported high dose opioid and high dose benzodiazepine/ alcohol use on at least one day. The latter group were more likely to use prescribed opioid substitution therapy, often alongside diverted pharmaceutical opioids. Socio-demographic and clinical profiles did not vary according to high dose opioid, alcohol and benzodiazepine use, and there was no association with harms. Conclusions Same-day use of opioids with benzodiazepines/alcohol, and high dose combinations, are common amongst people who tamper with pharmaceutical opioids. Assessment of concomitant benzodiazepine/alcohol use during opioid therapy, implementation of real-time prescription monitoring systems, and research to clarify upper safe limits for polydrug depressant use, are potential implications.
\end{abstract}

\section{Keywords}

pharmaceutical, opioids:, retrospective, 7-day, diary, study, who, people, amongst, depressants, system, nervous, central, other, opioids, tamper, same-day

\section{Disciplines}

Education | Social and Behavioral Sciences

\section{Publication Details}

Peacock, A., Bruno, R., Larance, B., Lintzeris, N., Nielsen, S., Ali, R., Dobbins, T. \& Degenhardt, L. (2016). Same-day use of opioids and other central nervous system depressants amongst people who tamper with pharmaceutical opioids: A retrospective 7-day diary study. Drug and Alcohol Dependence, 166 125-133.

\section{Authors}

Amy Peacock, Raimondo Bruno, Briony K. Larance, Nicholas Lintzeris, Suzanne Nielsen, Robert Ali, Timothy Dobbins, and Louisa Degenhardt 
Same-Day Use of Opioids and other Central Nervous System Depressants amongst People who Tamper with Pharmaceutical Opioids: A Retrospective 7-Day Diary Study

Amy Peacock ${ }^{1,2^{*}}$, Raimondo Bruno ${ }^{2}$, Briony Larance ${ }^{1}$, Nick Lintzeris ${ }^{3,4}$, Suzanne Nielsen ${ }^{1,4}$, Robert Ali $^{5}$, Timothy Dobbins ${ }^{1}, \&$ Louisa Degenhardt ${ }^{1}$

${ }^{1}$ National Drug and Alcohol Research Centre, University of New South Wales, Sydney, NSW, 2052, Australia

${ }^{2}$ School of Medicine, University of Tasmania, Tasmania, 7001, Australia ${ }^{3}$ Sydney Medical School, Sydney University, NSW, Australia

${ }^{4}$ Drug and Alcohol Services, South East Sydney Local Health District, NSW, Australia ${ }^{5}$ University of Adelaide, South Australia, Australia

Target journal: Drug and Alcohol Dependence

Link to publishers version http://www.drugandalcoholdependence.com/article/S0376$\underline{\text { 8716(16)30199-5/abstract }}$ 
Corresponding author:

*Amy Peacock, National Drug and Alcohol Research Centre, University of New South Wales, NSW, 2052. Email: Amy.Peacock@unsw.edu.au fax: +61 (02) 93850222

\section{Abstract}

Objective: The aims were to determine: i) quantity and frequency of same-day use of opioids with benzodiazepines and/or alcohol amongst people who regularly tamper with pharmaceutical opioids; and iii) socio-demographic, mental health, harms and treatment profile associated with same-day use of high doses.

Method: The cohort $(\mathrm{n}=437)$ completed a retrospective 7-day diary detailing opioid, benzodiazepine, and alcohol intake. Oral morphine equivalent (OME) units and diazepam equivalent units (DEU) were calculated, with $>200 \mathrm{mg}$ OME, $>40 \mathrm{mg}$ DEU and $>4$ standard alcoholic drinks (each 10g alcohol) considered a "high dose".

Results: One-half (47\%) exclusively consumed opioids without benzodiazepines/alcohol; $26 \%$ had days of opioid use with and without benzodiazepines/alcohol; and $26 \%$ always used opioids and benzodiazepines/alcohol. Same-day use of opioids with benzodiazepines/alcohol typically occurred on 1-3 days in the past week. Six in ten (61\%) participants reported high dose opioid use on at least one day; one in five (20\%) reported high dose opioid and high dose benzodiazepine/alcohol use on at least one day. The latter group were more likely to use prescribed opioid substitution therapy, often alongside diverted pharmaceutical opioids. Socio-demographic and clinical profiles did not vary according to high dose opioid, alcohol and benzodiazepine use, and there was no association with harms.

Conclusions: Same-day use of opioids with benzodiazepines/alcohol, and high dose combinations, are common amongst people who tamper with pharmaceutical opioids. Assessment of concomitant benzodiazepine/alcohol use during opioid therapy, 
implementation of real-time prescription monitoring systems, and research to clarify upper safe limits for polydrug depressant use, are potential implications.

Keywords: opioid; benzodiazepine; alcohol; concurrent use; polydrug abuse; overdose 


\section{Introduction}

There has been an increase in opioid prescriptions, partly attributable to greater chronic noncancer pain $(\mathrm{CNCP})$ prevalence and a greater number of opioids registered for $\mathrm{CNCP}$ treatment (Berterame et al., 2016; Karanges et al., 2016). Increased pharmaceutical availability has seen greater prevalence of extra-medical use and tampering (i.e., manipulating a dosage form to change its drug delivery in a way not specified by the manufacturer, and may include dissolving, crushing, chewing, snorting and injecting) (Katz et al., 2011; Katz et al., 2007), which in turn has led to greater rates of opioid-related harms (Hall et al., 2008; Laxmaiah Manchikanti et al., 2012).

Use of other central nervous system depressants, such as benzodiazepines and alcohol, with opioids may have an interactive or synergistic effect, increasing the risk of acute harms such as overdose (Warner-Smith et al., 2001; White and Irvine, 1999). An increasing proportion of unintentional overdoses have been observed involving the use of benzodiazepines, alcohol and other depressant drugs with opioids (Calcaterra et al., 2013; Jones et al., 2013), with death primarily attributed to respiratory depression (Gudin et al., 2013). As tolerance to the depressant effects of opioids is slower than tolerance to euphoric effects, consumers with a long history of opioid use are still often unknowingly at risk, although the underlying pathophysiology is complex and poorly understood (White and Irvine, 1999).

In addition, combined depressant use may contribute to increased sedation, greater motor impairment (e.g., falls or injuries) and enhanced abuse liability of these substances. Benzodiazepine or alcohol use enhances the euphoric effects of opioid analgesics (Chen et al., 2011; Lintzeris et al., 2007; Zacny and Gutierrez, 2011) and acute alcohol coadministration may increase maximum plasma concentrations and reduce time to maximum 
concentration for long-acting opioids (Fiske et al., 2012; Johnson et al., 2012; Sathyan et al., 2008) .

Few studies have conducted in-depth investigation of concurrent use (specifically, same-day use) of opioids and benzodiazepines and/or alcohol among people who use opioids (e.g., Brands et al., 2008; Cropsey et al., 2015; Fleming et al., 2007; Lavie et al., 2009; Nielsen et al., 2007; Saunders et al., 2012). This is particularly the case for people regularly engaging in extra-medical pharmaceutical opioid use, a group at greater risk of opioid-related harms and among whom use of a range of substances is prevalent (Becker et al., 2008; Jones, 2013). As such, the aims of this study were to apply a novel methodology, a retrospective self-report daily drug diary, to determine:

1. Frequency of same-day opioid use with benzodiazepines/alcohol, and typical quantities consumed, by people who regularly tamper with prescription opioids; and

2. Demographic, mental/physical health, drug use, harms and treatment profile correlates of same-day use of opioids, benzodiazepines, and alcohol at high doses. 


\section{Material and Methods}

\subsection{Participants and Procedure}

We used data from the National Opioid Medication Abuse Deterrence (NOMAD) study, a prospective cohort $(n=606)$ of people who reported regular extra-medical use and tampering with pharmaceutical opioids. Participants were recruited from metropolitan areas of New South Wales, Tasmania, and South Australia. Inclusion criteria required that participants: i) were aged $\geq 18$, ii) reported monthly or more frequent extra-medical pharmaceutical opioid use in the preceding six months, and iii) reported injecting, snorting, chewing or smoking pharmaceutical opioids on a monthly or more frequent basis in the preceding six months. Exclusion criteria comprised use of pharmaceutical opioids only in accordance with professional medical advice, or residing outside the metropolitan area/in prison for a month or longer in the preceding six months. Further details of the study are available from Degenhardt et al. (2015). The study was approved by the Human Research Ethics Committee of the University of New South Wales.

The current study utilised data from the third interview ('Wave 3') in April-June, 2015 $(n=499,82 \%$ of the original cohort; $90 \%$ of those not in residential treatment or prison at the time of interview, and still alive at the time of follow-up). Only data collected in the 60minute computer-assisted interview from participants who completed the seven-day retrospective drug diary component ( $\mathrm{n}=5$ excluded) and who reported at least one day of opioid use ( $n=57$ excluded) were included (final sample n=437; see Supplementary Table 1 for comparison of final sample versus those excluded).

\subsection{Key Measures}

\subsubsection{Drug Diary}


Using a Timeline Follow-back approach (Sobell and Sobell, 1996), participants were asked to identify any alcohol, illicit drugs, and prescription medicines (including those not directly prescribed to them) which they had consumed in the past seven days. Participants reported intake on each day from the day prior to interview (Day 1) to Day 7. The approximate time of waking and sleeping (and time of drug consumption where reported) were obtained to facilitate recall. Participants reported the type/brand, quantity, unit (e.g., four 40mg morphine tablets), route of administration, and source (e.g., own prescription, bought from dealer). Prompt cards with photographs of medications (full array of tablet sizes) were used to help identify the correct medicine/formulation.

\subsubsection{Sample Characteristics and Mental/Physical Health}

In addition to standard socio-demographic items, depression and generalised anxiety disorder were measured using the PHQ-9 and GAD-7 modules of the Patient Health Questionnaire (Kroenke et al., 2010). Likely presence of moderate to severe depression was defined as a PHQ-9 score of $\geq 10$ (Kroenke et al., 2001); and likely symptoms of moderate to severe anxiety were defined as a GAD-7 score of $\geq 10$ (Spitzer et al., 2006). Participants were also asked the first item of the Brief Pain Inventory; specifically, whether they had experienced pain (non-everyday) in the past month (Tan et al., 2004).

\subsubsection{Drug Use and Behaviours}

The Composite International Diagnostic Interview (World Health Organisation, 2001) assessed past-12 month pharmaceutical opioid use disorder based on the International Classification of Diseases (ICD-10) criteria. Participants reported whether they had used heroin, cannabis and methamphetamine in the past month. A short form of the Alcohol Use Disorders Identification Test (AUDIT-C) was included; a cut-off score of 5 was used to 
indicate alcohol-related problems (Bush et al., 1998). The Severity of Dependence Scale (SDS) was used to assess severity of benzodiazepine dependence; a cut-off score of 7 was used to indicate possible dependence (Cuevas et al., 2000).

\subsubsection{Harms and Treatment}

Participants were asked about frequency of injecting any drug in the past month (with subsequent coding of binary 'any injection' and 'daily injection' variable), and whether they had overdosed in the past 12 months. Participants also reported past month engagement in opioid substitution treatment (OST) and past 12 month hospital and general practitioner visits (for any health-related reason).

\subsection{Data Analysis}

\subsubsection{Calculating Oral Morphine Equivalent (OME) Units and Diazepam Equivalent Units} $(\mathrm{DEU})$

Opioid doses reported in the daily diary were converted into oral morphine equivalent (OME) units using conversion factors outlined by Nielsen et al. (2015). This conversion allows for comparison of opioid consumption across participants using different opioids based on the concept of equianalgesic dosing, where different doses of different opioids have similar analgesic effects. An OME unit is measured as being equivalent to $1 \mathrm{mg}$ of orallyadministered morphine. The conversion factors have been created such that the OME consumed on a particular day is calculated by multiplying the quantity of the particular opioid by the appropriate factor accounting for route of administration; for example, a participant who injected $80 \mathrm{mg}$ of oxycodone on one day has consumed 240mg OME (80mg multiplied by conversion unit for oxycodone injection of 3). 
A similar process was undertaken to calculate diazepam equivalent units (DEU) (National Prescribing Service, 1990; The Royal Australian College of General Practitioners, 2015; Therapeutic Guidelines Limited, 2013). One DEU is equivalent to 1mg of orallyadministered diazepam. The number of DEU consumed on a particular day was calculated by multiplying the quantity of the particular benzodiazepine by the appropriate factor (e.g., oral $0.5 \mathrm{mg}$ alprazolam is multiplied by conversion unit of 10$)$. Conversion rates for non-oral route of administration were not used although they would be anticipated to be higher (only 8 participants reported injecting benzodiazepines in the past month).

\subsubsection{Statistical Analyses}

SPSS Statistics Version 22 (IBM, Somers, NY) was used to calculate descriptive statistics, including percentages for categorical outcomes and median and interquartile range (M, IQR) for continuous outcomes. The percentage of participants who reported each opioid consumption practice (opioid only, same-day opioid and benzodiazepine, same-day opioid and alcohol, and same-day opioid, benzodiazepine and alcohol use) across the seven days; percentage of participants reporting 0-7 days of each consumption practice; and the proportion of opioid use days where each of these consumption practices were reported for daily opioid users, were calculated.

Median days of use and intake were reported for each consumption practice, as well as the percentage of participants exceeding a high dose threshold on any day of use of that consumption practice. The cut-off for a high opioid dose was $>200 \mathrm{mg}$ OME based on previously determined cut-offs of opioid dose associated with high overdose risk (Manchikanti et al., 2012). The cut-off for a high benzodiazepine dose was $>40 \mathrm{mg}$ DEU reflecting the upper maximum daily dose of oral diazepam licensed in Australia (National 
Prescribing Service, March 2016). In previous laboratory studies, 40mg oral diazepam (but not 10 or $20 \mathrm{mg}$ ) was associated with features of respiratory depression in combination with routine methadone doses in a proportion of patients in methadone treatment (Lintzeris et al., 2006; Lintzeris et al., 2007). The high alcohol dose cut-off was $>4$ standard drinks (1 standard drink $=10 \mathrm{~g}$ ) in accordance with Australian National Health and Medical Research Council guidelines to minimise risk of acute harms (National Health and Medical Research Council, 2009).

Multinomial logistic regression analyses were conducted to identify the socio-demographic, mental and physical health, drug use, harms and treatment characteristics associated with: i) opioid use $\leq 200 \mathrm{mg}$ OME on every day of use, ii) opioid use $>200 \mathrm{mg}$ OME on at least one day of opioid use [referent category], and iii) opioid use $>200 \mathrm{mg}$ and same-day use of benzodiazepine $>40 \mathrm{mg}$ and/or alcohol intake $>4$ standard drinks on at least one day. Relative risk ratios (RRR) are reported.

Concern has been expressed regarding conversion of oral methadone to oral morphine equivalents using a linear dose conversion (Anderson et al., 2001), as large methadone doses (e.g. $>80 \mathrm{mg}$ ) translates to very high OME (e.g. $>300 \mathrm{mg}$ ). Further, there is evidence of reduced mortality risk with long-term methadone maintenance dosing (Cornish et al., 2010). Consequently, sensitivity analyses were also run for frequency and intake estimates, and correlates of high dose intake, excluding those participants who had used only prescribed opioid substitution therapy (OST) only (including those who reported prescribed OST and prescribed pharmaceutical opioid use) due to concerns that conversion rates for methadone may be artificially inflating OME dose. 


\section{Results}

\subsection{Sample Characteristics}

At Wave 3, the majority of the sample were male (66\%), with a median age of 43 (IQR 3649). Half (57\%) reported income $<$ AUD $\$ 400 /$ week. Over one-tenth were currently homeless $(14 \%)$, and $63 \%$ reported ever being incarcerated. Based on data provided in the interview, $60 \%(n=263)$ had received opioid substitution treatment (OST); 36\% had used their own prescribed benzodiazepines; 59\% had used any benzodiazepines (all sources); and 48\% had used alcohol, in the past month. The majority of the sample $(85 \%)$ had injected a drug in the past month (34\% daily injection), and two-thirds (67\%) met ICD-10 criteria for an opioid use disorder in the preceding 12 months.

\subsection{Frequency and quantity of same-day use}

The majority of participants (70\%) reported opioid use every day in the preceding week; $9 \%$ reported 6 days of opioid use, $10 \%$ reported $4-5$ days, $7 \%$ reported $2-3$ days, and only $5 \%$ reported one day of opioid use (Figure 1). Indeed, on a given day, a maximum of $17 \%$ reported no opioid use, $61 \%$ opioid only use, $21 \%$ opioid and benzodiazepine use, $10 \%$ opioid and alcohol use, and 6\% opioid, benzodiazepine and alcohol use (Supplementary Figure 1).

One-half of the sample (47\%) consumed exclusively only opioids only throughout the week; one quarter (26\%) had day(s) where they consumed opioids alone and day(s) where they consumed opioids and benzodiazpines and/or alcohol; and 26\% reported that all days of opioid use also involved benzodiazepines and/or alcohol. Of note, $38 \%$ of the latter group alternated between same-day use of opioids and benzodiazepines, same-day use of opioids and alcohol, and/or same-day use of opioids, benzodiazepines and alcohol. 
Examination of the number of days participants engaged in each opioid consumption behaviour showed that $34 \%$ of the sample reported one or more days of same-day opioid and benzodiazepine use ( $8 \%$ daily use); $19 \%$ reported one or more days of same-day opioid and alcohol use (3\% daily use); and $11 \%$ reported one or more days of same-day opioid, benzodiazepine and alcohol use (1\% daily use) (Figure 1).

***Figure 1 approximately here***

Focusing specifically on the $70 \%(n=306)$ of participants who reported using opioids every day in the past week, same-day use of opioids with benzodiazepines and/or alcohol was infrequent (Supplementary Figure 2). One-third (37\%) reported reported using opioids and benzodiazepines on the same day ( $25 \%$ of these reported only one day of such use), $20 \%$ reported using opioids and alcohol on the same day ( $42 \%$ of these reported only one day of such use), and $11 \%$ reported using opioids, benzodiazepines and alcohol the same day (50\% of these reported only one day of such use).

Median OME when consuming opioids only (i.e., without benzodiazepines or alcohol on the same day) was 380mg (Table 1); 400mg OME when using opioids and benzodiazepines; 410mg OME when using opioid, benzodiazepine and alcohol use; and 353mg OME when using opioids and alcohol. Considerable variability in typical intake was evident given the interquartile range for estimates (Table 1). When considering dose on any day of opioid use (i.e., regardless of whether benzodiazepine or alcohol ingested), $80 \%$ of the sample reported at least one day where they exceeded 200mg OME.

***Table 1 approximately here*** 
Median benzodiazepine dose was similar for those reporting same-day opioid and benzodiazepine use with and without alcohol (both 20mg DEU), with $30 \%$ and $26 \%$ exceeding 40mg DEU on at least one day, respectively. Median alcohol intake was higher when participants reported same-day opioid and alcohol use versus same day opioid, benzodiazepine and alcohol use (4.6 versus 3.3 standard drinks), with $61 \%$ and $53 \%$ exceeding four standard alcoholic drinks on at least one day, respectively (Table 1).

\subsection{Socio-Demographic, Mental and Physical Health, Drug Use, Harms and Treatment Characteristics of Each Group}

Participants were classified into:

1. 'High Dose Opioid Group': reported using >200mg OME on at least one day (but no same-day use of $>40 \mathrm{mg}$ DEU and/or > four standard alcoholic drinks; 61\%, $\mathrm{n}=267$ );

2. 'Lower Dose Opioid Group': reported only using $\leq 200 \mathrm{mg}$ OME (20\%, n=89); and

3. 'High Dose Combined Depressant Group': one-fifth $(20 \%, \mathrm{n}=81)$ reported:

a. $\geq 1$ day of using $>200 \mathrm{mg}$ OME and $>40 \mathrm{mg}$ DEU $(8 \% ; n=33)$;

b. $\geq 1$ day of using $>200 \mathrm{mg}$ OME and $>4$ standard alcoholic drinks $(10 \%, n=42)$;

c. Both $3 \mathrm{~A}$ and $3 \mathrm{~B}$ on separate days $(1 \%, \mathrm{n}=3)$; and

d. $\geq 1$ day of using $>200 \mathrm{mg}$ OME, $>40 \mathrm{mg}$ DEU and $>4$ standard alcoholic drinks $(1 \%, n=3)$.

Breakdown of these three groups according to use of prescribed, non-prescribed and illicit opioids (Table 2) showed that the majority (53\%) of the 'Lower Dose Opioid Group' had consumed exclusively non-prescribed or illicit opioids in the preceding seven days, while only one-fifth of the 'High Dose Opioid Group' (22\%) and the 'High Dose Combined Depressant Group' (17\%) reported such use. Four-fifths of the 'High Dose Opioid Group' (78\%) and the 'High Dose Combined Depressant Group' (83\%) had received pharmaceutical 
opioids and/or OST from a medical professional, with $38 \%$ and $35 \%$ using exclusively prescribed opioids in the preceding week, respectively.

The 'High Dose Combined Depressant Group' had a greater RRR of depression than the 'High Dose Opioid Group' (Table 3). There were similar rates of use of illicit drugs, daily injection and overdose across the groups, although low numbers should be noted in the latter case. The 'High Dose Combined Depressant Group' had a greater RRR of past month cannabis use and prescribed benzodiazepine use relative to the 'High Dose Opioid Group', with $48 \%$ of the former group reporting prescribed benzodiazepine use.

\subsection{Sensitivity Analyses}

Sensitivity analyses excluding those who used prescribed OST only (final subsample $n=301$ ) showed that whilst median OME decreased, the percentage of participants who reported exceeding the opioid high dose threshold for each opioid consumption practice was similar relative to the full sample (Supplementary Table 2). Median DEU and standard drink intake, and the percentage who exceeded high dose thresholds for these substances when also using opioids, were similar in the full sample and subsample. Approximately two-thirds of the 'High Dose Opioid Group' (66\%, n=175) and the 'High Dose Combined Depressant Group' $(69 \%, n=56)$, and four-fifths $(79 \%, n=70)$ of the 'Lower Dose Opioid Group', were retained in the subsample, suggesting that removal of those who used prescribed OST only did not result in a decrement in the high opioid dose groups only (Supplementary Table 3). Identification of correlates of group was hampered by low statistical power, although there was a trend towards higher rates of depression amongst the 'High Dose Combined Depressant Group' relative to 'High Dose Opioid Group', as per analysis of the full sample. 


\section{Discussion}

This study is unique relative to previous research examining opioid, benzodiazepine and alcohol use (e.g., Brands et al., 2008; Cropsey et al., 2015; Fleming et al., 2007; Lavie et al., 2009; Nielsen et al., 2007; Saunders et al., 2012) given the depth of data on frequency and quantity of use. Over two-thirds of the current sample had used opioids every day in the preceding week, and over half reported at least one day where they used opioids and benzodiazepines/alcohol. The majority of participants engaged in this consumption practice infrequently, and only one-tenth reported same-day use of all three. Whilst one-fifth of the sample consistently used $\leq 200 \mathrm{mg}$ OME ('Lower Dose Opioid Group'), three-fifths used $>200 \mathrm{mg}$ OME on one or more days ('High Dose Opioid Group') and one-fifth used >200mg OME plus $>40 \mathrm{mg}$ DEU and/or $>4$ standard alcoholic drinks on at least one day ('High Dose Combined Depressant Group'). Opioid intake for the 'Lower Dose Opioid Group’ was primarily derived from non-prescribed or illicit opioids use, whereas half of the 'High Dose Combined Depressant Group' had used both prescribed pharmaceutical opioids/OST and non-prescribed/illicit opioids in the preceding week. However, rates of exceeding the high dose threshold were maintained when excluding participants who used prescribed OST only. There were few statistically-significant group differences in demographics, health, substance use, harms and treatment, with the exception that the 'High Dose Combined Depressant Group' had greater risk of depression (consistent with findings of Rooney et al., 1999; Ross and Darke, 2000) and past month cannabis use.

The finding that same-day use of opioids with benzodiazepines and/or alcohol is common amongst the sample fits with prior research (Fleming et al., 2007; Saunders et al., 2012), with the exception of lower rates of concurrent use amongst criminal justice samples (Cropsey et 
al., 2015). However, it is the general infrequency of this behaviour, and evidence of variability in use of benzodiazepines and/or alcohol alongside opioids from day to day, that is of concern in regards to adverse events. The elevated risk of overdose from use of benzodiazepines, independently and with other depressants, is well-established (Buykx et al., 2010; Darke et al., 1996). Repeated administration of psychoactive substances results in tolerance, whereby the effects of a substance are reduced due to neuroadaptation. However, in the absence of regular use there may be less tolerance to the sedating effects of benzodiazepines/alcohol, resulting in a greater risk of respiratory depression and subsequent overdose (Jones et al., 2012; Lintzeris and Nielsen, 2010). In this study, greater risk of nonfatal overdose was not evident amongst the group reporting ingesting high opioid doses with same-day high benzodiazepine/alcohol intake. However, this sample comprised individuals who are generally ingesting high opioid doses (>200mg OME) on a daily basis, and caution should be applied in interpreting these findings due to low statistical power (i.e., smaller group sizes coupled with low rate of overdose).

\subsection{Implications}

Prescribing guidelines recommend medical supervision in opioid therapy and OST; concurrent prescribing of benzodiazepines is generally not recommended or, where unavoidable, used only under certain conditions (e.g., lower doses of methadone or when prescribing buprenorphine; Manchikanti et al., 2012; Nielsen et al., 2007). Concomitant consumption of alcohol with opioids is not advised (Gowing et al., 2014; Health et al., 2009). However, four-fifths of the 'High Dose Combined Depressant Group' had used pharmaceutical opioids and/or OST prescribed by a medical professional in the past week, and half had received prescribed benzodiazepines in the preceding month. Consequently, it appears that the majority of those at risk of harm are already under supervision of a 
prescribing health professional. It is not clear if the OST prescriber would be aware of benzodiazepines being prescribed by other prescribers, or alcohol consumption unless specifically screened for. Implementation of real-time prescription monitoring across states and territories in Australia may rectify this situation to some extent (Ogeil et al., 2016). However, it will not address the risk associated with concomitant benzodiazepine use due to the exclusion of most benzodiazepines from planned monitoring programs in Australia (Nielsen and Bruno, 2014). Nor will it identify use of diverted pharmaceutical opioids, which represents the majority of non-prescribed pharmaceutical opioid use in this group. Notably, half of the high dose opioid groups were using opioids sourced outside the bounds of a doctor's prescription. This raises important questions about how unsanctioned medication use is best detected above and beyond comprehensive assessment by prescribers, and the role of regular urine drug screening in the prescribing of opioids (both in the context of chronic noncancer pain and OST) (Ross et al., 2007; Starrels et al., 2010). Further, the role of harm reduction services in education may also be critical as those people using unsanctioned medications may be unlikely to discuss the risks with health professionals for fear of disclosure adversely affecting their supply. Take-home naloxone provision where there is risk of combined depressant use might be an important precautionary action in such cases.

The three groups were not distinguishable on the basis of screening measures for opioid dependence, benzodiazepine dependence, and hazardous alcohol use. This suggests that these substance-specific screening questionnaires may not discriminate those at risk of adverse outcomes for using concomitant opioids with other CNS depressants. The three groups were also not distinguishable on the basis of past year overdose and other harms, potentially due to low rates of these outcomes. These findings reinforce the need to extend beyond screening for dependence or aberrant medication-related behaviours to explicitly gathering information 
regarding concomitant benzodiazepine and alcohol use when prescribing opioid therapy or OST, with possible implementation of those measures mentioned above (e.g., urine drug testing, provision of naloxone) to increase the possibility that hazardous depressant use is detected and harms are prevented where self-report is not accurate. Comprehensive assessment of overdose risk factors, such as concomitant depressant use, via both self-report and objective measures is critical to minimise behaviours and ensure harm reduction measures are considered.

Review of guidelines for this manuscript yielded various maximum recommended opioid doses to reduce overdose risk (typically ranging between 40-200mg OME) (Dowell et al., 2016; Manchikanti et al., 2012). Guidelines as to the maximum benzodiazepine and alcohol intake concomitant with opioids were not available, and recommendations for benzodiazepine prescribing typically focused on maximum dose for therapeutic purpose as opposed to the threshold for adverse outcomes. A lack of consensus or even specification of high dose thresholds most likely reflects a lack of understanding and scarcity of data regarding polydrug interactions. Further to this, the current analyses presume a set cut-off for each individual. Whilst not possible to take this into consideration in the current study, each individual's risk threshold will vary according to their history of use of the substances, as well as individual factors such as physical health and changes in tolerance. Again, these findings highlight the importance of a comprehensive assessment of risk factors for adverse outcomes when prescribing opioids.

\subsection{Limitations}

While the methodology provided considerable depth of detail regarding same-day patterns of use, it is important to note that this sample does not generalise to all opioid consumers. 
Participants entered the study on the basis of extra-medical use and tampering with pharmaceutical opioids, and over half were receiving OST, meaning that this sample could theoretically already be identified as high risk. This conclusion is reinforced by the majority exceeding the high opioid dose (from heroin, pharmaceutical opioids and/or OST intake) on any day in the preceding week.

Data were retrospectively self-reported however participants were provided in-depth instruction with multiple prompts; there were no negative consequences for disclosing behaviours; interviewers were trained; and previous research points to validity and reliability of self-report in assessing drug use (Darke, 1998), particularly when using a Timeline Follow-back approach (Hjorthøj et al., 2012). It should also be noted that a conservative approach to data collection was undertaken, whereby failure to recall use that day was marked as abstinent.

Published equivalence tables taking into account route of administration were used for conversion to standard units (OME and DEU). Whilst issues around conversion of methadone dose to OME have been flagged, caution should also be applied when considering conversion ratios in general, being influenced by individual factors (e.g., opioid exposure, bioavailability, and presence of other drugs), particularly chronic consumption (Patanwala et al., 2007; Pereira et al., 2001). Conversion for opioid dose took into account oral versus intravenous administration, however these analyses also do not account for the role of route of administration and associated overdose risk (Brugal et al., 2002; Degenhardt et al., 2011).

\subsection{Conclusion}


This cohort comprised a diverse group of participants who report regular use of high doses of a range of prescribed, diverted and illicit opioids. Same-day use of opioids with benzodiazepines and/or alcohol was common, but did not typically occur daily, and there were different patterns of concomitant use of alcohol and/or benzodiazepines (e.g., use of opioids and benzodiazepines on one day, and opioids and alcohol on the next). This type of use poses high risk for adverse outcomes due to relatively lower tolerance, although participants in the current study reporting high dose same-day depressant use were not distinguishable from those using high opioid doses only in regards to rate of overdose. Most of those reporting same-day high opioids doses with high benzodiazepine/alcohol doses used opioids dispensed by a medical, speaking to the importance of explicit assessment of concomitant depressant use when prescribing and monitoring treatment, potentially faciliated by urine drug sceen and real-time prescription monitoring. Further, research assessing safety thresholds for concomitant depressant use is essential given a lack of consensus regarding, or even data to inform, upper safe limits for each drug. 


\section{References}

Anderson, R., Saiers, J.H., Abram, S., Schlicht, C., 2001. Accuracy in Equianalgesic Dosing: Conversion Dilemmas. Journal of Pain Symptom Management 21, 397-406.

Becker, W.C., Sullivan, L.E., Tetrault, J.M., Desai, R.A., Fiellin, D.A., 2008. Non-medical use, abuse and dependence on prescription opioids among US adults: Psychiatric, medical and substance use correlates. Drug and Alcohol Dependence 94, 38-47.

Berterame, S., Erthal, J., Thomas, J., Fellner, S., Vosse, B., Clare, P., Hao, W., Johnson, D.T., Mohar, A., Pavadia, J., 2016. Use of and barriers to access to opioid analgesics: a worldwide, regional, and national study. The Lancet 387, 1644-1656.

Brands, B., Blake, J., Marsh, D.C., Sproule, B., Jeyapalan, R., Li, S., 2008. The impact of benzodiazepine use on methadone maintenance treatment outcomes. Journal of Addictive Disorders 27, 37-48.

Brugal, M.T., Barrio, G., Fuente, L.D.L., Regidor, E., Royuela, L., Suelves, J.M., 2002. Factors associated with non-fatal heroin overdose: assessing the effect of frequency and route of heroin administration. Addiction 97, 319-327.

Bush, K., Kivlahan, D.R., McDonell, M.B., Fihn, S.D., Bradley, K.A., for the Ambulatory Care Quality Improvement, P., 1998. The audit alcohol consumption questions (audit-c): An effective brief screening test for problem drinking. Archives of Internal Medicine $158,1789-1795$.

Buykx, P., Loxley, W., Dietze, P., Ritter, A., 2010. Medications used in overdose and how they are acquired - an investigation of cases attending an inner Melbourne emergency department. Australian and New Zealand Journal of Public Health 34, 401-404.

Calcaterra, S., Glanz, J., Binswanger, I.A., 2013. National trends in pharmaceutical opioid related overdose deaths compared to other substance related overdose deaths: 1999-2009. Drug and Alcohol Dependence 131, 263-270. 
Chen, K.W., Berger, C.C., Forde, D.P., D'Adamo, C., Weintraub, E., Gandhi, D., 2011. Benzodiazepine use and misuse among patients in a methadone program. BMC Psychiatry $11,90$.

Cornish, R., Macleod, J., Strang, J., Vickerman, P., Hickman, M., 2010. Risk of death during and after opiate substitution treatment in primary care: prospective observational study in UK General Practice Research Database. British Medical Journal 341.

Cropsey, K.L., Stevens, E.N., Valera, P., Clark, C.B., Bulls, H.W., Nair, P., Lane, P.S., 2015. Risk factors for concurrent use of benzodiazepines and opioids among individuals under community corrections supervision. Drug and Alcohol Dependence 154, 152-157.

Cuevas, C.D.L., Sanz, E.J., Padilla, J., Berenguer, J.C., 2000. The Severity of Dependence Scale (SDS) as screening test for benzodiazepine dependence: SDS validation study. Addiction 95, 245-250.

Darke, S., 1998. Self-report among injecting drug users: a review. Drug and Alcohol Dependence 51, 253-263.

Darke, S., Ross, J., Hall, W., 1996. Overdose among heroin users in Sydney, Australia: I. Prevalence and correlates of non-fatal overdose. Addiction 91, 405-411.

Degenhardt, L., Bucello, C., Mathers, B., Briegleb, C., Ali, H., Hickman, M., McLaren, J., 2011. Mortality among regular or dependent users of heroin and other opioids: a systematic review and meta-analysis of cohort studies. Addiction 106, 32-51.

Degenhardt, L., Larance, B., Bruno, R., Lintzeris, N., Ali, R., Farrell, M., 2015. Evaluating the potential impact of a reformulated version of oxycodone upon tampering, nonadherence and diversion of opioids: the National Opioid Medications Abuse Deterrence (NOMAD) study protocol. Addiction 110, 226-237.

Dowell, D., Haegerich, T.M., Chou, R., 2016. CDC guideline for prescribing opioids for chronic pain-United States, 2016. Journal of the Americal Medical Association. 
Fiske, W.D., Jobes, J., Xiang, Q., Chang, S.-C., Benedek, I.H., 2012. The Effects of Ethanol on the Bioavailability of Oxymorphone Extended-Release Tablets and Oxymorphone Crush-Resistant Extended-Release Tablets. The Journal of Pain 13, 90-99.

Fleming, M.F., Balousek, S.L., Klessig, C.L., Mundt, M.P., Brown, D.D., 2007. Substance use disorders in a primary care sample receiving daily opioid therapy. The Journal of Pain 8, 573-582.

Gowing, L., Ali, R., Dunlop, A., Farrell, M., Lintzeris, N., 2014. National guidelines for medication-assisted treatment of opioid dependence. Commonwealth of Australia, Canberra, 38-39.

Gudin, J.A., Mogali, S., Jones, J.D., Comer, S.D., 2013. Risks, management, and monitoring of combination opioid, benzodiazepines, and/or alcohol use. Postgraduate <edicine 125, 115-130.

Hall, A.J., Logan, J.E., Toblin, R.L., Kaplan, J.A., Kraner, J.C., Bixler, D., Crosby, A.E., Paulozzi, L.J., 2008. Patterns of abuse among unintentional pharmaceutical overdose fatalities. Journal of the American Medical Association 300, 2613-2620.

Health, W.H.O.D.o.M., Abuse, S., Organization, W.H., Board, I.N.C., Drugs, U.N.O.o., Crime, 2009. Guidelines for the psychosocially assisted pharmacological treatment of opioid dependence. World Health Organization.

Hjorthøj, C.R., Hjorthøj, A.R., Nordentoft, M., 2012. Validity of Timeline Follow-Back for self-reported use of cannabis and other illicit substances — Systematic review and metaanalysis. Addictive Behaviors 37, 225-233.

Johnson, F.K., Ciric, S., Boudriau, S., Kisicki, J., Stauffer, J., 2012. Effects of Alcohol on the Pharmacokinetics of Morphine Sulfate and Naltrexone Hydrochloride Extended Release Capsules. The Journal of Clinical Pharmacology 52, 747-756. 
Jones, C.M., 2013. Heroin use and heroin use risk behaviors among nonmedical users of prescription opioid pain relievers - United States, 2002-2004 and 2008-2010. Drug and Alcohol Dependence 132, 95-100.

Jones, C.M., Mack, K.A., Paulozzi, L.J., 2013. Pharmaceutical overdose deaths, United States, 2010. Journal of the American Medical Association 309, 657-659.

Jones, J.D., Mogali, S., Comer, S.D., 2012. Polydrug abuse: a review of opioid and benzodiazepine combination use. Drug and Alcohol Dependence 125, 8-18.

Karanges, E.A., Blanch, B., Buckley, N.A., Pearson, S.A., 2016. Twenty-five years of prescription opioid use in Australia: A whole-of-population analysis using pharmaceutical claims. British Journal of Clinical Pharmacology.

Katz, N., Dart, R.C., Bailey, E., Trudeau, J., Osgood, E., Paillard, F., 2011. Tampering with prescription opioids: nature and extent of the problem, health consequences, and solutions. The American Journal of Drug and Alcohol Abuse 37, 205-217.

Katz, N.P., Adams, E.H., Chilcoat, H., Colucci, R.D., Comer, S.D., Goliber, P., Grudzinskas, C., Jasinski, D., Lande, S.D., Passik, S.D., 2007. Challenges in the development of prescription opioid abuse-deterrent formulations. The Clinical Journal of Pain 23, 648660.

Kroenke, K., Spitzer, R., Williams, J.B.W., 2001. The PHQ-9: Validity of a Brief Depression Severity Measure. Journal of General Internal Medicine 16, 606-613.

Kroenke, K., Spitzer, R.L., Williams, J.B.W., Lowe, B., 2010. The Patient Health Questionnaire Somatic, Anxiety, and Depressive Symptom Scales: a systematic review. General Hospital Psychiatry 32, 345-359.

Lavie, E., Fatséas, M., Denis, C., Auriacombe, M., 2009. Benzodiazepine use among opiatedependent subjects in buprenorphine maintenance treatment: correlates of use, abuse and dependence. Drug and Alcohol Dependence 99, 338-344. 
Laxmaiah Manchikanti, M., Standiford Helm, I., MA, J.W.J., PhD, V.P., MSc, J.S.G., DO, P., 2012. Opioid epidemic in the United States. Pain Physician 15, 2150-1149.

Lintzeris, N., Mitchell, T.B., Bond, A., Nestor, L., Strang, J., 2006. Interactions on mixing diazepam with methadone or buprenorphine in maintenance patients. Journal of Clinical Psychopharmacology 26, 274-283.

Lintzeris, N., Mitchell, T.B., Bond, A.J., Nestor, L., Strang, J., 2007. Pharmacodynamics of diazepam co-administered with methadone or buprenorphine under high dose conditions in opioid dependent patients. Drug and Alcohol Dependence 91, 187-194.

Lintzeris, N., Nielsen, S., 2010. Benzodiazepines, methadone and buprenorphine: interactions and clinical management. The American Journal on Addictions 19, 59-72.

Manchikanti, L., Abdi, S., Atluri, S., Balog, C.C., Benyamin, R.M., Boswell, M.V., Brown, K.R., Bruel, B.M., Bryce, D.A., Burks, P.A., 2012. American Society of Interventional Pain Physicians (ASIPP) guidelines for responsible opioid prescribing in chronic noncancer pain: Part 2--guidance. Pain Physician 15, S67-116.

National Health and Medical Research Council, 2009. Australian guidelines to reduce health risks from drinking alcohol. National Health and Medical Research Council, Canberra.

National Prescribing Service, 1990. National Prescribing Service Newsletter: Helping patients withdraw from benzodiazepines.

http://www.nps.org.au/_data/assets/pdf file/0020/15761/news04 benzodiazepines 069 9.pdf.accessed on.

National Prescribing Service, March 2016. Valium tablets. http://www.nps.org.au/medicines/brain-and-nervous-system/anxietymedicines/diazepam/valium-tablets accessed on 20th May 2016.

Nielsen, S., Bruno, R., 2014. Implementing real-time prescription drug monitoring: Are we ready? Drug andAalcohol Review 33, 463-465. 
Nielsen, S., Degenhardt, L., Hoban, B., Gisev, N., 2015. A synthesis of oral morphine equivalents (OME) for opioid utilisation studies Pharmacoepidemiology and Drug Safety.

Nielsen, S., Dietze, P., Lee, N., Dunlop, A., Taylor, D., 2007. Concurrent buprenorphine and benzodiazepines use and self-reported opioid toxicity in opioid substitution treatment. Addiction 102, 616-622.

Ogeil, R.P., Heilbronn, C., Lloyd, B., Lubman, D., 2016. Prescription drug monitoring in Australia: capacity and coverage issues. Medical Journal of Australia 204, 148.

Patanwala, A.E., Duby, J., Waters, D., Erstad, B.L., 2007. Opioid conversions in acute care. Annals of Pharmacotherapy 41, 255-267.

Pereira, J., Lawlor, P., Vigano, A., Dorgan, M., Bruera, E., 2001. Equianalgesic dose ratios for opioids: a critical review and proposals for long-term dosing. Journal of Pain Symptom Management 22, 672-687.

Rooney, S., Kelly, G., Bamford, L., Sloan, D., O'connor, J., 1999. Co-abuse of opiates and benzodiazepines. Irish Journal of Medical Science 168, 36-41.

Ross, E.L., Janfaza, D., Nedeljkovic, S.S., Narang, S., 2007. Urine toxicology screening among chronic pain patients on opioid therapy: frequency and predictability of abnormal findings. Clinical Journal of Pain 23, 173-179.

Ross, J., Darke, S., 2000. The nature of benzodiazepine dependence among heroin users in Sydney, Australia. Addiction 95, 1785-1793.

Sathyan, G., Sivakumar, K., Thipphawong, J., 2008. Pharmacokinetic profile of a 24-hour controlled-release OROS formulation of hydromorphone in the presence of alcohol. Current Medical Research and Opinion 24, 297-305.

Saunders, K., Von Korff, M., Campbell, C.I., Banta-Green, C.J., Sullivan, M.D., Merrill, J.O., Weisner, C., 2012. Concurrent Use of Alcohol and Sedatives among Persons 
Prescribed Chronic Opioid Therapy: Prevalence and Risk Factors. The Journal of Pain $13,266-275$.

Sobell, L.C., Sobell, M.B., 1996. Timeline followback user's guide: A calendar method for assessing alcohol and drug use. Toronto: Addiction Research Foundation.

Spitzer, R.L., Kroenke, K., Williams, J.B.W., Lowe, B., 2006. A brief measure for assessing generalized anxiety disorder: the GAD-7. Archives of Internal Medicine 166, 1092-1097.

Starrels, J.L., Becker, W.C., Alford, D.P., Kapoor, A., Williams, A.R., Turner, B.J., 2010. Systematic review: treatment agreements and urine drug testing to reduce opioid misuse in patients with chronic pain. Annals of Internal Medicine 152, 712-720.

Tan, G., Jensen, M.P., Thornby, J.I., Shanti, B.F., 2004. Validation of the Brief Pain Inventory for chronic nonmalignant pain. Journal of Pain 5, 133-137.

The Royal Australian College of General Practitioners, 2015. Prescribing drugs of dependence in general practice, Part B - Benzodiazepines. The Royal Australian College of General Practitioners, Melbourne.

Therapeutic Guidelines Limited, 2013. eTG complete: Psychotropic Guidelines. http://etg.tg.com.au/complete/ accessed on.

Warner-Smith, M., Darke, S., Lynskey, M., Hall, W., 2001. Heroin overdose: causes and consequences. Addiction 96, 1113-1125.

White, J.M., Irvine, R.J., 1999. Mechanisms of fatal opioid overdose. Addiction 94, 961-972.

World Health Organisation, 2001. Composite International Diagnostic Interview World Health Organisation, Geneva.

Zacny, J.P., Gutierrez, S., 2011. Subjective, psychomotor, and physiological effects of oxycodone alone and in combination with ethanol in healthy volunteers. Psychopharmacology 218, 471-481. 


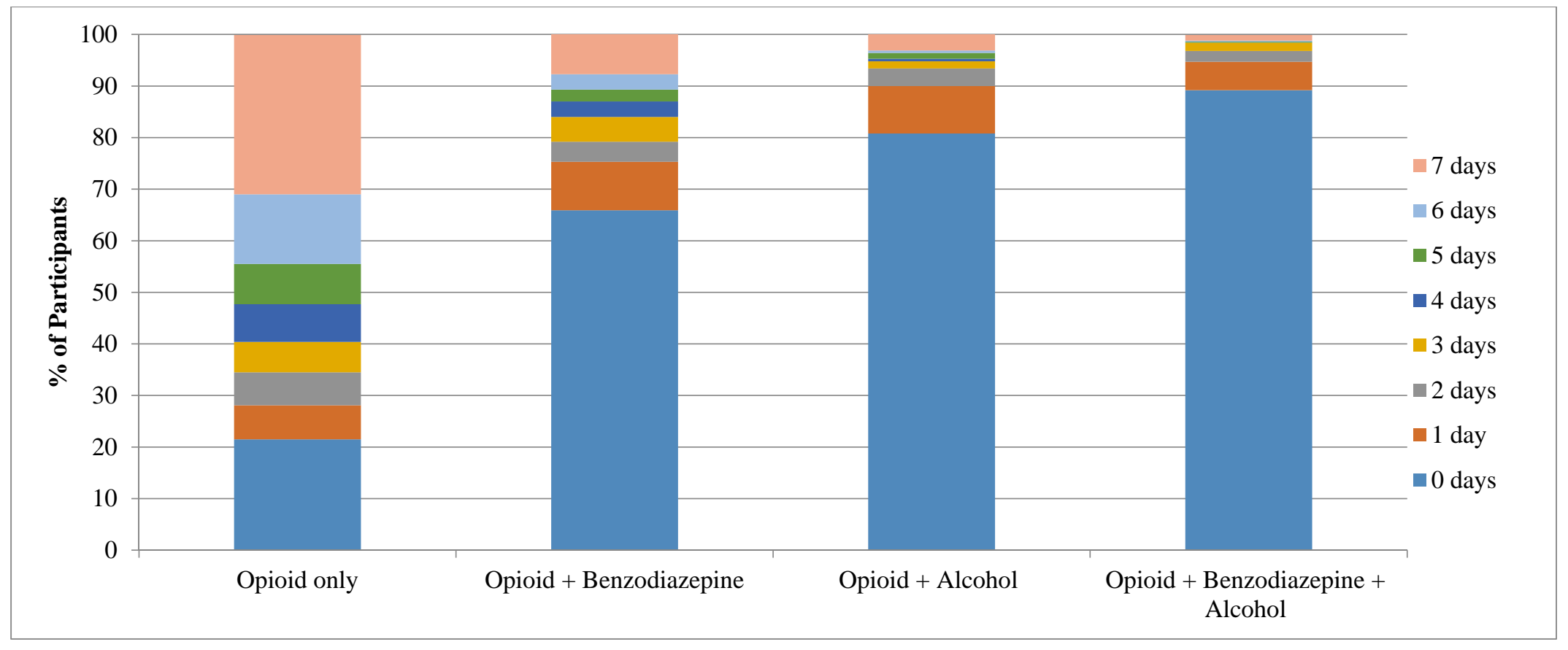

Figure 1. Percentage of participants according to number of days in the past week (0-7 days) self-reported opioid use, with and without benzodiazepine and alcohol use. 
Table 1

Frequency and Quantity of Opioid Use, with and without Benzodiazepines and Alcohol

\begin{tabular}{|c|c|c|c|c|c|c|c|c|}
\hline $\begin{array}{l}\text { Same-Day } \\
\text { Consumption } \\
\text { Practice }\end{array}$ & $\begin{array}{c}\text { Total sample } \\
\text { reporting at } \\
\text { least one day } \\
\text { of behaviour } \\
\%(n)\end{array}$ & $\begin{array}{c}\text { Median } \\
\text { Days of } \\
\text { Use (IQR) }\end{array}$ & $\begin{array}{c}\text { Median mg } \\
\text { OME/Day } \\
\text { (IQR) }\end{array}$ & $\begin{array}{c}\text { \% > 200mg } \\
\text { OME/Day } \\
\text { (on any day of } \\
\text { use) }\end{array}$ & $\begin{array}{c}\text { Median mg } \\
\text { DEU/Day (IQR) }\end{array}$ & $\begin{array}{c}\%>40 \mathrm{mg} \\
\text { DEU/Day (any } \\
\text { day) }\end{array}$ & $\begin{array}{c}\text { Median } \\
\text { Standard } \\
\text { Drinks/Day } \\
\text { (IQR) }\end{array}$ & $\begin{array}{c}\text { \% >4 Standard } \\
\text { Drinks/Day } \\
\text { (any day) }\end{array}$ \\
\hline $\begin{array}{l}\text { Any opioid use } \\
\text { (regardless of } \\
\text { concurrent use) }\end{array}$ & 100 (437) & $7(6-7)$ & 390 (196-621) & 80 & - & - & - & - \\
\hline Opioid only & 79 (343) & $6(4-7)$ & 380 (199-621) & 79 & - & - & - & - \\
\hline $\begin{array}{l}\text { Opioid + } \\
\text { Benzodiazepine }\end{array}$ & 34 (149) & $3(1-6)$ & $400(180-595)$ & 79 & $20(10-34)$ & 26 & - & - \\
\hline Opioid + Alcohol & $19(84)$ & $2(1-4.75)$ & 353 (182-559) & 69 & - & - & $4.6(3.0-9.8)$ & 61 \\
\hline
\end{tabular}

Note. All participants report at least one day of opioid use; possible range for days of use is 1-7. Note that the any opioid use results represent findings for any days on which opioids are used, regardless of whether benzodiazepine or alcohol use is present or absent. The median dose for the remaining outcomes (opioid only, opioid + benzodiazepine, opioid + alcohol, opioid +benzodiazepine + alcohol) is only calculated for days of use for the specific behaviour (e.g., median OME and DEU across days having opioids + benzodiazepines); and the percentage exceeding the cut-off (200mg OME, 40mg DEU, 4 standard alcoholic drinks) is calculated based on dose on any day where that consumption practice is reported ('any day'). OME: oral morphine equivalent units; DEU: oral diazepam equivalent units; IQR: interquartile range. 
Table 2

Percentage Reporting Prescribed, Non-Prescribed and Illicit Opioid Use According to Group

\begin{tabular}{|c|c|c|c|c|}
\hline & $\begin{array}{c}\text { Total Sample } \\
\qquad \mathbf{n}=437\end{array}$ & $\begin{array}{c}\text { A. } \\
\text { High Dose } \\
\text { Opioid Group } \\
\text { n=267 }\end{array}$ & $\begin{array}{c}\text { B. } \\
\text { Lower Dose } \\
\text { Opioid Group } \\
\text { n=89 }\end{array}$ & $\begin{array}{c}\text { C. } \\
\text { High Dose Opioid and } \\
\text { Benzodiazepine/Alcohol } \\
\text { Group } \\
\text { n=81 }\end{array}$ \\
\hline$\%$ prescribed PO only & 7 & 3 & 21 & 4 \\
\hline$\%$ prescribed OST only & 29 & 32 & 20 & 30 \\
\hline $\begin{array}{l}\% \text { prescribed PO and OST } \\
\text { use only }\end{array}$ & 2 & 3 & 1 & 1 \\
\hline $\begin{array}{l}\text { \% prescribed PO and } \\
\text { diverted/illicit }\end{array}$ & 8 & 9 & 3 & 9 \\
\hline $\begin{array}{l}\text { \% prescribed OST and } \\
\text { diverted/illicit }\end{array}$ & 26 & 31 & 1 & 35 \\
\hline $\begin{array}{l}\text { \% prescribed PO and } \\
\text { prescribed OST and non- } \\
\text { prescribed/illicit }\end{array}$ & 2 & 1 & - & 5 \\
\hline \% non-prescribed/illicit only & 27 & 22 & 53 & 17 \\
\hline
\end{tabular}

Note. Diverted/illicit refers to any use of non-prescribed pharmaceutical opioids, non-prescribed OST, and/or heroin. PO: pharmaceutical opioid (morphine, oxycodone, tramadol, physeptone, Norspan, fentanyl, codeine); OST: opioid substitution treatment (methadone syrup, Subutex, Suboxone). 
Table 3

Correlates of High Dose Opioid Use, With and Without High Dose Alcohol and/or Benzodiazepine Concurrent Use (N=437)

\begin{tabular}{|c|c|c|c|c|c|c|c|}
\hline & $\begin{array}{c}\text { Total Sample } \\
\text { N }\end{array}$ & $\begin{array}{c}\text { Total Sample } \\
\% \text { (95\%CI) }\end{array}$ & $\begin{array}{c}\text { A. } \\
\text { High Dose } \\
\text { Opioid Group } \\
\text { N=267 } \\
\text { \% (95\%CI) }\end{array}$ & $\begin{array}{c}\text { B. } \\
\text { Lower Dose } \\
\text { Opioid Group } \\
\text { N=89 } \\
\text { \% (95\%CI) }\end{array}$ & $\begin{array}{c}\text { C. } \\
\text { High Dose } \\
\text { Combined } \\
\text { Depressant } \\
\text { Group } \\
\text { N=81 } \\
\%(95 \% \mathrm{CI})\end{array}$ & $\begin{array}{c}\text { B vs A (ref) } \\
\text { RRR (95\%CI) }\end{array}$ & $\begin{array}{c}\text { C vs A (ref) } \\
\text { RRR (95\%CI) }\end{array}$ \\
\hline \multicolumn{8}{|l|}{ Demographics } \\
\hline Age (M, IQR)\# & 436 & $43(36-49)$ & $43(35-49)$ & $42(37-48)$ & $42(36-49)$ & $1.01(0.98-1.03)$ & $1.01(0.98-1.03)$ \\
\hline Male & 437 & 66 & 66 & 67 & 67 & $1.07(0.64-1.78)$ & $1.03(0.61-1.75)$ \\
\hline Unemployed (B) & 437 & 82 & 82 & 79 & 83 & $0.79(0.43-1.43)$ & $1.02(0.53-1.97)$ \\
\hline Education <year 10 (B) & 436 & 27 & 26 & 27 & 30 & $1.05(0.61-1.81)$ & $1.20(0.69-2.08)$ \\
\hline Income $<\$ 400$ per week & 434 & 48 & 47 & 50 & 53 & $1.15(0.71-1.86)$ & $0.79(0.48-1.30)$ \\
\hline Homeless/no fixed abode & 436 & 14 & 13 & 17 & 15 & $1.38(0.71-2.68)$ & $1.27(0.77-2.09)$ \\
\hline Lifetime prison history & 437 & 63 & 62 & 58 & 70 & $0.87(0.53-1.42)$ & $1.47(0.86-2.51)$ \\
\hline \multicolumn{8}{|l|}{ Mental/Physical Health } \\
\hline $\begin{array}{l}\text { Pain (non-everyday) past } \\
\text { month }\end{array}$ & 435 & 57 & 55 & 60 & 58 & $1.19(0.73-1.94)$ & $1.10(0.66-1.82)$ \\
\hline
\end{tabular}




\begin{tabular}{|c|c|c|c|c|c|c|c|}
\hline $\begin{array}{l}\text { Moderate/severe depression } \\
(\geq 10 \text { PHQ- } 9)\end{array}$ & 420 & 48 & 45 & 47 & 58 & $1.09(0.66-1.78)$ & $1.71(1.03-2.84)^{*}$ \\
\hline $\begin{array}{l}\text { Moderate/severe anxiety }(\geq 10 \\
\text { GAD-7) }\end{array}$ & 417 & 33 & 33 & 23 & 41 & $0.61(0.34-1.09)$ & $1.41(0.84-2.37)$ \\
\hline \multicolumn{8}{|l|}{ Drug Use and Behaviours } \\
\hline $\begin{array}{l}\text { ICD-10 opioid use disorder } \\
\text { (past } 12 \text { months) }\end{array}$ & 432 & 67 & 65 & 72 & 66 & $1.42(0.83-2.42)$ & $1.06(0.63-1.80)$ \\
\hline $\begin{array}{l}\text { Benzodiazepine dependence } \\
\text { (SDS score } \geq 7 \text { ) }\end{array}$ & 432 & 11 & 11 & 7 & 18 & $0.63(0.25-1.57)$ & $1.80(0.89-3.61)$ \\
\hline $\begin{array}{l}\text { Risky alcohol consumption } \\
\text { (AUDIT-C score } \geq 5 \text { ) }\end{array}$ & 436 & 72 & 68 & 76 & 77 & $1.50(0.86-2.60)$ & $1.51(0.85-2.68)$ \\
\hline Used cannabis past month & 436 & 65 & 61 & 66 & 79 & $1.26(0.76-2.08)$ & $2.36(1.31-4.26)^{* *}$ \\
\hline Used heroin past month & 437 & 48 & 49 & 42 & 49 & $0.74(0.45-1.20)$ & $1.01(0.62-1.67)$ \\
\hline $\begin{array}{l}\text { Used methamphetamine past } \\
\text { month }\end{array}$ & 437 & 47 & 45 & 47 & 52 & $1.10(0.68-1.77)$ & $1.32(0.80-2.17)$ \\
\hline \multicolumn{8}{|l|}{ Harms and Treatment } \\
\hline Injected any drug past month & 436 & 85 & 85 & 82 & 90 & $0.83(0.44-1.57)$ & $1.66(0.75-3.71)$ \\
\hline Injected daily past month & 436 & 34 & 38 & 27 & 27 & $0.59(0.35-1.01)$ & $0.60(0.35-1.04)$ \\
\hline Overdose past $12 \mathrm{~m}$ & 435 & 9 & 9 & 8 & 10 & $0.86(0.36-2.07)$ & $1.12(0.48-2.60)$ \\
\hline $\begin{array}{l}\text { Used prescribed } \\
\text { benzodiazepine past month }\end{array}$ & 437 & 36 & 31 & 39 & 48 & $1.44(0.87-2.36)$ & $2.06(1.24-3.42)^{* *}$ \\
\hline $\begin{array}{l}\text { Hospital visit past } 12 \mathrm{~m} \text { (for any } \\
\text { health-related reason) }\end{array}$ & 433 & 10 & 10 & 9 & 14 & $0.90(0.39-2.08)$ & $1.46(0.69-3.10)$ \\
\hline $\begin{array}{l}\text { Seen GP past } 12 \mathrm{~m} \text { (for any } \\
\text { health-related reason) }\end{array}$ & 433 & 72 & 71 & 65 & 80 & $0.76(0.45-1.26)$ & $1.62(0.88-2.97)$ \\
\hline
\end{tabular}

Note. Statistically significant values $(\mathrm{p}<.050)$ are bolded. (B) These outcomes were assessed in the Wave 1 interview; all other items were assessed in Wave 3. PHQ-9: Patient Health Questionnaire; GAD-7: Generalized Anxiety Disorder 7-item scale; ICD10: International

Classification of Diseases version 10; SDS: Severity of Dependence Scale; AUDIT-C: Alcohol Use Disorders Identification Test-C; GP: general 
practitioner; 12m: 12 months; M: median; IQR: interquartile range; RRR: relative risk ratio; 95\%CI: 95\% confidence interval.*p<.050; $* * p<.010$. 